\title{
Evaluation of Patients with Blunt Abdominal Injury by Ultrasonography (USG) and Multi Detector Computed Tomography (MDCT)
}

\author{
P. Madhu' ${ }^{1}$ D. Santhosh ${ }^{2}$ \\ ${ }^{1}$ Associate Professor, ${ }^{2}$ Assistant Professor: Department of Radiodiagnosis, Government Medical College, Nizamabad, \\ Telangana. India
}

Corresponding author: Dr. D. Santhosh, MBBS, MD (Radiodiagnosis), Department of Radiodiagnosis, Government Medical College, Nizamabad, Telangana. India.

DOI: http://dx.doi.org/10.21276/ijcmsr.2019.4.4.42

How to cite this article: P. Madhu, D. Santhosh. Evaluation of patients with blunt abdominal injury by ultrasonography (USG) and multi detector computed tomography (MDCT). International Journal of Contemporary Medicine Surgery and Radiology. 2019;4(4):D177-D180.

\section{A B S T R A C T}

Introduction: Computed tomography (CT) used in cases of blunt abdominal trauma has been found sensitive in detection of bowel and mesenteric injuries and discrimination of operable from nonoperable candidates. Study aimed to evaluate the role of USG \& MDCT in identifying IAI in patients with BAT and to provide information that could accurately determine choice of management.

Material and methods: It was a prospective study done in the department of radiology done for a period of 1 year from September 2018 to September 2019. Evaluation of 50 patients with blunt abdominal injury by USG and MDCT. They were subjected to USG followed by MDCT.

Results: Most of the patients admitted to the hospital were victims of RTAs (33 cases) accounting for $66 \%$ of blunt abdominal trauma. The age group of patients was very wide, ranging from 5-80 years, most commonly affected age group is $21-40$ yrs.32(64\%). Males are more affected than females, Male:Female ratio was 42:8. Most common affected organs were liver and spleen, accounting for $33 \%$ and $26 \%$ respectively. Third common affected organ was Hollow viscus (23\%). Hemoperitoneum was observed in 28 patients. Sensitivity of USG in detecting hemoperitoneum was $89.28 \%$. Sensitivity of MDCT in detecting hemoperitoneum was $100 \%$. Sensitivity of USG in detecting solid organ injuries was $78.57 \%$. Sensitivity of MDCT in detecting solid organ injuries was $92.3 \%$.

Conclusion: MDCT is the superior diagnostic modality in the diagnosis of blunt abdominal trauma. USG can be a valuable initial investigation.

Keywords: Blunt Abdominal Injury, Ultrasonography (USG), Multi Detector Computed Tomography (MDCT)

\section{INTRODUCTION}

Abdominal trauma is one of the commonest injuries. Blunt abdominal trauma (BAT) usually occurs due to road traffic accidents (RTA), fall from heights or during sports. Prevalence of intraabdominal injury (IAI) varies widely, ranging from $7.7 \%$ to $65 \% .{ }^{1}$ The Indian fatality rates for trauma are 20 times that for developed countries. About 30\% of such deaths are thought to be preventable. ${ }^{3}$ Rapid diagnosis is essential and appropriately prioritizing diagnostic work up and treatment is critical to ensure patient survival. Although diagnostic peritoneal lavage (DPL) is thought to be superior to clinical examination in assessing abdominal injuries, it is an invasive procedure.

$\mathrm{CT}$ of the abdomen can depict such injuries accurately and is relatively noninvasive. CT as the sole modality enables evaluation of other associated injuries in addition to global evaluation of abdominal trauma in hemodynamically stable patients and is extensively used in North American centers as the initial modality of diagnosis. ${ }^{2}$ During past several years, USG has become an important modality in many centers in the screening of BAT.USG is the primary imaging modality of choice for diagnosis of IAI. It is non invasive, rapid, relatively inexpensive and a reliable diagnostic tool for assessment of presence of abdominal fluid and in detecting liver, spleen and kidney injuries.

The purpose of this study was to evaluate the role of USG and MDCT in identifying IAI in patients with BAT and to provide information that could accurately determine choice of management (non-operative vs operative).

\section{MATERIAL AND METHODS}

It was a prospective study done in the department of radiology on 50 cases with history of blunt abdominal trauma for a period of 1 year from September 2018 to September 2019.

\section{Inclusion criteria}

All the patients irrespective of age and sex referred to the 
Department of Radiology and Imaging with history of blunt abdominal trauma.

\section{Exclusion criteria}

Patients who were hemodynamically unstable, Pregnant women and Penetrating injuries.

USG examinations were performed with a curvilinear and linear probe on Samsung ultrasound machine. The presence of free fluid within the abdominal cavity was accepted as a positive sign for hemoperitoneum. Visceral organs were evaluated for parenchymal abnormalities consisting of intraparenchymal hematomas, lacerations, and/or geographic zones of echotextural heterogeneity.

USG examination was followed by MDCT examination. MDCT examinations were carried out on 16 Slice MDCT Siemens Somatom Emotion 6. Free fluid with attenuation value $>30$ Hounsfield Units $(\mathrm{HU})$ was labeled as hemoperitoneum. Injuries to individual organs were graded according to organ injury scaling (OIS) system and injury severity grades.

The decision to manage patients either conservatively or proceed to laparotomy was made by the attending surgeon based on clinical condition along with USG and MDCT results. USG findings were compared with the findings

\begin{tabular}{|l|c|}
\hline Age group in years & $\begin{array}{c}\text { Number of } \\
\text { patients n } \\
\text { (Percentage) }\end{array}$ \\
\hline $01-20$ & $07(14 \%)$ \\
\hline $21-40$ & $32(64 \%)$ \\
\hline $41-60$ & $08(16 \%)$ \\
\hline $61-80$ & $03(06 \%)$ \\
\hline Gender & \\
\hline Male & $42(84 \%)$ \\
\hline Female & $08(16 \%)$ \\
\hline Mode of injury & \\
\hline RTA & $33(66 \%)$ \\
\hline fall from heights & $05(10 \%)$ \\
\hline automobile vs pedestrian & $05(10 \%)$ \\
\hline assault & $03(6 \%)$ \\
\hline $\begin{array}{l}\text { others(bull horn injury, hit by a projectile } \\
\text { object) }\end{array}$ & $04(8 \%)$ \\
\hline \multicolumn{2}{|l|}{ Table-1: Demographic distribution in present study (N-50) } \\
\hline
\end{tabular}

obtained by MDCT and laparotomy. MDCT was used as the diagnostic standard. In cases where surgery was done, surgical findings were taken as the standard. Patients were followed up until they were discharged from the hospital. Sensitivity, specificity, positive predictive value and negative predictive value of USG and MDCT were calculated.

Technique for CT study: Risk of contrast administration were explained to the patients and informed consent was obtained prior to the contrast study. Routine anteroposterior topogram of the abdomen was initially taken in all patients in the supine position. Axial sections of $5 \mathrm{~mm}$ thickness was taken from the level of lung bases to the level of ischial tuberosities. Kilovolt peak:120-140 kvp, Milliampere second:200-250mAs for an average sized patient (increased values for oversized patients).Pitch:1.5,Field of view:240$350 \mathrm{~mm}$;Collimation:2.5mm, Matrix size:512x512,Time for scan:4-5 seconds.

Plain scans were followed by intravenous contrast scans. For intravenous contrast enhancement, $80-100 \mathrm{ml}$ of dynamic injection of (Iversol-300 $\mathrm{mg}$ of iodine per $\mathrm{ml}$ ) or in children a dose of $300 \mathrm{mg}$ of iodine $/ \mathrm{Kg}$ body wt was administered and axial sections were taken. Sections were taken in arterial (30sec) and portal venous (60-90sec) phases. Delayed scanning (5-7min) was not routinely performed, only in suspected cases of renal or bladder traumas. Post study reconstructions were done at $2.5 \mathrm{~mm}$. Sagittal and Coronal reconstructions were made wherever necessary. The scans were viewed on a direct display console at multiple window settings (i.e abdomen window at 320/40; Lung window at 1400/-600; Bone window of 2400/200).

AAST classification of pancreatic injury, liver injury, splenic injury and renal injury were considered to read CT scan.

\section{RESULTS}

Majority of patients were of 21-40 yr age group (64\%). Most common of the patients were men (84\%). Most common mode of injury was RT. (66\%) (table-1).

A total of 43 organ injuries were noted in 38 patients. Most common injured organ is Liver(33\%). 6 cases showed multiorgan injuries (fig-1).

FP- 2cases of suspected liver laceration on USG were not seen on MDCT. FN-4cases of organ injuries were not detected on USG. 2 cases of hemoperitoneum were not

\begin{tabular}{|l|c|c|c|}
\hline \multirow{2}{*}{ USG } & \multicolumn{3}{|c|}{ MDCT } \\
\cline { 2 - 4 } & Positive for intra abdominal injury & negative for intra abdominal injury & Total \\
\hline Positive for intra abdominal injury & $32(\mathrm{TP})$ & $2(\mathrm{FP})$ & 34 \\
\hline Negative for intra abdominal injury & $6(\mathrm{FN})$ & $10(\mathrm{TN})$ & 16 \\
\hline Total & 38 & 12 & 50 \\
\hline \multicolumn{2}{|r|}{ Table-2: Comparison of USG diagnosis with MDCT diagnosis (N-50) } \\
\hline
\end{tabular}

\begin{tabular}{|l|c|c|c|}
\hline USG & \multicolumn{3}{|c|}{ Operative } \\
\hline & Positive for solid organ injury & Negative for solid organ injury & Total \\
\hline Positive for solid organ injury & $11(\mathrm{TP})$ & $02(\mathrm{FP})$ & 13 \\
\hline Negative for solid organ injury & $03(\mathrm{FN})$ & $05(\mathrm{TN})$ & 08 \\
\hline Total & 14 & 07 & 21 \\
\hline \multicolumn{4}{|l|}{ Table-3: Comparison of USG diagnosis with operative diagnosis in solid organ injuries (n-21) } \\
\hline
\end{tabular}




\begin{tabular}{|l|c|c|c|}
\hline \multirow{2}{*}{ MDCT } & \multicolumn{3}{|c|}{ Operative } \\
\cline { 2 - 4 } & Positive for organ injury & Negative for organ injury & Total \\
\hline Positive for organ injury & $24(\mathrm{TP})$ & $01(\mathrm{FP})$ & 25 \\
\hline Negative for organ injury & $02(\mathrm{FN})$ & $03(\mathrm{TN})$ & 05 \\
\hline Total & 26 & 04 & 30 \\
\hline \multicolumn{2}{|}{ Table-4: Comparison of MDCT diagnosis with operative diagnosis (n-30) } \\
\hline
\end{tabular}

\begin{tabular}{|l|c|c|c|}
\hline \multirow{2}{*}{ USG } & \multicolumn{3}{|c|}{ MDCT } \\
\cline { 2 - 4 } & Positive for hemoperitoneum & Negative for hemoperitoneum & Total \\
\hline Positive for hemoperitoneum & $25(\mathrm{TP})$ & 02(FP) & 27 \\
\hline Negative for hemoperitoneum & $03(\mathrm{FN})$ & $20(\mathrm{TN})$ & 23 \\
\hline Total & 28 & 22 & 50 \\
\hline \multicolumn{2}{|r}{ Table-5: Comparison of USG diagnosis with MDCT diagnosis in hemoperitoneum (n-50) } \\
\hline
\end{tabular}

\begin{tabular}{|l|c|c|}
\hline & $\begin{array}{c}\text { Atif latif et al } \\
\text { (n-70) 2008 }\end{array}$ & $\begin{array}{c}\text { Present study } \\
\text { (n-50) }\end{array}$ \\
\hline Sensitivity & $93.3 \%$ & $84.21 \%$ \\
\hline Specificity & $85 \%$ & $83.33 \%$ \\
\hline Positive predictive value & $82.3 \%$ & $94.11 \%$ \\
\hline negative predictive value & $94.4 \%$ & $62.5 \%$ \\
\hline \multicolumn{2}{|c|}{ Table-6: Comparative study of role of USG in detection of } \\
intraabdominal injury \\
\hline
\end{tabular}

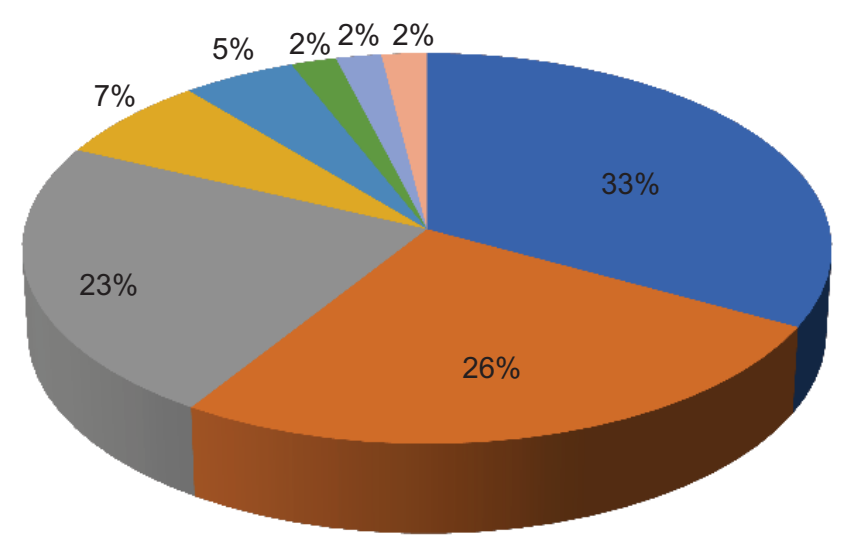

\begin{tabular}{|c|c|c|}
\hline Liver & Bowel & Mesentery \\
\hline Spleen & Kidney & Pancreas \\
\hline
\end{tabular}

Figure-1: Distribution of organ injuries

detected on USG (table-2).

Sensitivity $(\mathrm{TP} / \mathrm{TP}+\mathrm{FN}) \times 100$

$=84.21 \%$

Specificity $(\mathrm{TN} / \mathrm{TN}+\mathrm{FP}) \mathrm{x} 100$

$=83.33 \%$

Positive Predictive Value $(\mathrm{TP} / \mathrm{TP}+\mathrm{FP}) \times 100=94.11 \%$

Negative Predictive Value $(\mathrm{TN} / \mathrm{TN}+\mathrm{FN}) \times 100=62.5 \%$

FP- 2 cases of suspected Liver laceration on USG were not seen intraoperatively. FN-3: 2cases of Splenic injuries and 1 case of Renal injury were not detected on USG (table-3).

Sensitivity (TP / TP + FN)x100

$=78.57 \%$

Specificity (TN / TN + FP) x100

$=71.42 \%$

Positive Predictive Value (TP / TP + FP) $\mathrm{x} 100=84.61 \%$

Negative Predictive Value $(\mathrm{TN} / \mathrm{TN}+\mathrm{FN}) \times 100=62.50 \%$

FP-1 ligamentum teres was mistaken for a liver laceration in one case. FN-2 cases of mesenteric tear were not detected on MDCT (table-4).
Sensitivity $(\mathrm{TP} / \mathrm{TP}+\mathrm{FN}) \times 100$

Specificity $(\mathrm{TN} / \mathrm{TN}+\mathrm{FP}) \times 100$

$=92.30 \%$

Positive Predictive Value (TP / TP + FP $) \times 100=96 \%$

Negative Predictive Value $(\mathrm{TN} / \mathrm{TN}+\mathrm{FN}) \times 100=60 \%$

FP:2 cases of Ascitic fluid were mistaken for Hemoperitoneum. FN:3 cases of Hemoperitoneum were not detected on USG as free fluid developed later on because of time gap between performing USG and MDCT (table-5).

Sensitivity (TP / TP + FN) x100

$=89.28 \%$

Specificity (TN / TN + FP)x100

$=90.90 \%$

Positive Predictive Value (TP / TP + FP) $\mathrm{x} 100=92.59 \%$

Negative Predictive Value $(\mathrm{TN} / \mathrm{TN}+\mathrm{FN}) \times 100=86.95 \%$

\section{DISCUSSION}

In the present study, 50 cases with H/o Blunt abdominal trauma were subjected to USG and MDCT over a period of 1 year from September 2018 to September 2019. In this study, the youngest patient was 5 years old, and the oldest was aged 75 years. The maximum percentage of patients (64\%) were in the age range of 21 to 40 years. Majority of these patients $(66 \%)$ were involved in motor vehicle accidents. Out of 50 patients in this study, $76 \%$ (38 patients) were positive for abdominal injury and 24\% (12 patients) were negative. Six patients sustained polytrauma with injuries involving more than one viscera or system. There was one death in this study. Out of the $76 \%$ (38 patients) who were positive for Intra abdominal injury, $73.7 \%$ (28 patients) had hemoperitoneum. In the present study majority of the cases were of 21-40 yr age group which is in accordance with the study done by Srivastava et al. ${ }^{2}$ The present study indicated male prevalence (84\%) which is in accordance with study by Atif Latif et al. ${ }^{1}$ The most frequent cause of Blunt abdominal injury in the present study was road traffic accident which is in accordance with a study by Srivastava et $\mathrm{al}^{2}$.

In the present study majority of the cases were of RTA which is in accordance with the study done by Srivastava et al. ${ }^{2}$ The present study showed Liver to be the most common injured organ which defers from the study done by Srivastava et al where Spleen was the most common injured organ.

Out of 34 positive cases on USG, 32 patients (94\%) were confirmed on MDCT as Intra abdominal injury. 14 patients had Liver injuries (33\%) and out of these eight had grade 2 injuries while six had grade 3 injuries. 11 patients had Splenic 
injuries (26\%) and out of these, six had grade 2 injuries, four had grade 3 and two had grade 5 injury. 10 cases of Hollow viscus perforation (23\%). 3 Renal injuries (7\%), 2 were of grade 3 and 1 of grade 4.

USG findings were compared to MDCT and laparotomy findings. MDCT correlated well with surgical findings in all 30 operated cases. In two cases, mesenteric tear was seen at laparotomy, which could not be detect at MDCT scan. MDCT results after comparison with operative findings revealed that 24 were true positive, 1 was false positive, 3 true negative, and 2 false negative. USG results after comparison with operative findings revealed that 11 were true positive, 2 were false positive, 5 true negative, and 3 false negative. Sensitivity and Specificity of USG in detection of intraabdominal injury in the present study correlated well with the study done by Atif Latif et al. ${ }^{1}$

In the present study USG detected free fluid in 27 patients. On MDCT 2 out of these 27 patients were labeled as negative for IAI (false positive) as free fluid was found to be ascitic fluid based on HU on MDCT. 3 cases of Hemoperitoneum were not detected on USG as free fluid developed later on as there was time gap between performing USG and MDCT. In a study by Richards et $\mathrm{al}^{4}$ on 744 patients, out of 51 patients who had free fluid identified by USG, 9 were false positive results; of these 9 patients 7 were female who had pelvic free fluid. Hence, most of these false positive results were reported to be originating from the physiological fluid observed in females. The OIS is a relatively new system with sole purpose to establish uniformity in different studies and thereby facilitate easy comparison.

Sensitivity and Specificity of USG in detection of intraabdominal injury in the present study correlated well with the study done by Atif Latif et $\mathrm{al}^{1}$ (table-6).

Kwashima et al.5 mentioned that multiorgan involvement occurs in $75 \%$ of those with blunt trauma; however, it differs regarding its association with penetrating trauma that he mentioned that it occurs in $80 \%$ of patients with penetrating trauma. This may be due to the fact that most patients with penetrating trauma included in this study had stab injury directed toward the flanks resulting in isolated renal injury. The most commonly injured intraabdominal organ associated with renal injury was the liver (46\%) followed by the spleen (30\%), and this is consistent with Ramchandani et al. who stated that the liver and the spleen are the most common intrabdominal organs to be injured with blunt trauma. ${ }^{6}$ Jeffrey et al. state that CT staging of blunt hepatic injuries has little discriminatory value in predicting outcome of stable patients, as nearly all have an excellent prognosis. ${ }^{7}$

Ilahi et al. in their study found that CT was 68\% (19 of 28) accurate in diagnosing pancreatic injury. ${ }^{8}$ They concluded that $\mathrm{CT}$ scan is only moderately sensitive and can underestimate or miss pancreatic injury.

Sclafani et al. consider CT the method of choice for renal injuries and confirmatory angiography unnecessary. ${ }^{9}$ Lupetin et al. using CT, diagnosed renal artery occlusion in all seven patients with renal trauma in their series..$^{10} \mathrm{He}$ mentioned that $\mathrm{CT}$ and angiography were equal in their usefulness for predicting the location of the injured portion of the renal arterial system in the four patients in whom preoperative angiography was performed. Although CT did not specifically show the point of obstruction in the renal artery or its branches that were shown angiographically, the location of the occlusion could be inferred by determining whether the distribution of the unenhanced parenchyma was total or segmental. CT showed retroperitoneal hematoma that was not visible on angiography in two cases. Angiography did not reveal any abnormalities that were not shown with $\mathrm{CT}$.

\section{CONCLUSION}

The present study concluded that MDCT is the superior diagnostic modality in the diagnosis of blunt abdominal trauma. USG can be a valuable initial investigation, however, USG can miss crucial injuries and may lead to inappropriate management in some patients. Hence it is imperative that all USG positive cases should be followed by MDCT. Similarly MDCT must also be performed in symptomatic patients with negative USG scans and in patients with suboptimal USG scans.

\section{REFERENCES}

1. Atif Latif, Muhammad Ashraf Farooq, Muhammad Adeel Azhar. Diagnostic Value of Ultrasonography in Evaluation of Blunt Abdominal Trauma. Rawal Med J 2008;33(1):154-159

2. Mallik K, Vashisht S, Thakur S, Srivastava DN. Comparative evaluation of ultrasonography and CT in patients with abdominal trauma: A prospective study. Indian J Radiol Imaging 2000; 10(3): 237-43

3. MM kumar, M.Venkataramanappa,I.Venkatrathnam et al. Prospective evaluation of Blunt abdominal trauma by CT.IJRI 2005;15(2):167-173.

4. John R. Richards, Nicolette A. Knopf, Lianyi Wang, John P. MaGahan Blunt abdominal trauma in children: Evaluation with emergency US. Radiology 2002;222(6): 749-754

5. A. Kawashima, C.M. Sandler, J.N. Corriere Jr., et al.:Ureteropelvic junction injuries secondary to blunt abdominal trauma: Radiology 1997;205(5):487-490.

6. P. Ramchandani,P.M. Buckler: Imaging of genitourinary trauma AJR Am J Roentgenol, 2009; 192(6):1514-1523

7. R.B. Jeffrey Jr. CT diagnosis of blunt hepatic and splenic injuries: a look to the future (editorial): Radiology, 1989;171(1):17-18.

8. O. Ilahi, G.V. Bochicchio, T.M. Scalea: Efficacy of computed tomography in diagnosis of pancreatic injury in adult blunt trauma patients: A single institutional study: Am Surg, 2002:68(3);704-708.

9. S.J. Sclafani, J.A. Becker, G.W. Shaftan, T.F. Phillips, A.S. Goldstein, J. Haller, et al. Strategies for the radiologic management of genitounnary trauma: Urol Radiol, 1985;7:231-244

10. A.R. Lupetin, B.L. Mainwanng, R.H. Daffner CT diagnosis of renal artery injury caused by blunt abdominal trauma: AJR 1989;153:1065-1068.

\section{Source of Support: Nil; Conflict of Interest: None}

Submitted: 21-10-2019; Accepted: 25-11-2019; Published online: 26-12-2019 